\title{
Suppressive Effect of Polyphenols from Immature Pear Fruits on Blood Glucose Levels
}

\author{
Zhaohong $\mathrm{Ci}^{1,2}$, Chengyu Jiang ${ }^{1,2}$, Yang Cui ${ }^{1}$, Michiyuki Kojima ${ }^{2,3, *}$ \\ ${ }^{1}$ Department of Food Science, Obihiro University of Agriculture and Veterinary Medicine, 11, \\ Nishi-2-sen, Inada-machi, Obihiro, Hokkaido 080-8555, Japan \\ ${ }^{2}$ United Graduate School of Agricultural Sciences, Iwate University, 3-18-8, Ueda, Morioka, Iwate 020-8550, Japan \\ ${ }^{3}$ Department of Human Sciences, Obihiro University of Agriculture and Veterinary Medicine, 11, Nishi-2-sen, \\ Inada-machi, Obihiro, Hokkaido 080-8555, Japan \\ *Corresponding author: kojima@obihiro.ac.jp
}

\begin{abstract}
The agricultural practice of pear thinning involves the removal of immature pear fruits, which are usually treated as waste but can contain high levels of beneficial polyphenols. To assess whether these fruits provide commercial utility, we aimed to examine the inhibitory effects of polyphenols from the immature pear fruits of the Hosui and Kosui cultivars on $\alpha$-glucosidase in vitro and blood glucose levels in mice after the oral administration of starch. Polyphenols were extracted from the immature pear fruits by $80 \%$ ethanol and $70 \%$ acetone, and the mixed extraction was purified by HP-20 column. The polyphenol contents of the Hosui and Kosui immature pear fruits were 28.5 and $20.9 \mathrm{mg} / \mathrm{g}$ D.W., respectively, and both were characterized by high percentages of oligomeric and polymeric polyphenols. The 2,2-diphenyl-1-picrylhydrazyl (DPPH) radical scavenging activities of the polyphenols from Hosui and Kosui fruits were 115.8 and $76.3 \mu \mathrm{mol} / \mathrm{g}$ D.W., respectively, indicating a significant difference between the two cultivars. Moreover, compared with the polyphenols from immature Kosui fruits, which had an $\mathrm{IC}_{50}$ value of $66.4 \mu \mathrm{g} / \mathrm{mL}$, those from Hosui fruits, with an $\mathrm{IC}_{50}$ value of $21.3 \mu \mathrm{g} / \mathrm{mL}$, resulted in more potent inhibition of $\alpha$-glucosidase activity. Polyphenols were fractionated with a Sephadex LH-20 column into fraction I (Fra.I; monomeric), fraction II (Fra.II; oligomeric), and fraction III (Fra.III; polymeric). Compared with Fra.I, Fra.II and Fra.III from immature Hosui and Kosui fruits showed higher inhibition of $\alpha$-glucosidase activity, with the highest inhibitory activity in Fra.III. Thus, $\alpha$-glucosidase inhibitory activity appears to be related to the degree of polyphenol polymerization. Moreover, polyphenols from immature Hosui fruits effectively suppressed increases in blood glucose levels after the oral administration of starch in mice. These results suggest that the immature pear fruits of the Hosui and Kosui cultivars have useful properties and, in particular, potential applications for the treatment of diabetes.
\end{abstract}

\section{Keywords: immature pear, polyphenol, DPPH radical scavenging activity, $\alpha$-glucosidase, blood glucose}

Cite This Article: Zhaohong Ci, Chengyu Jiang, Yang Cui, and Michiyuki Kojima, "Suppressive Effect of Polyphenols from Immature Pear Fruits on Blood Glucose Levels." Journal of Food and Nutrition Research, vol. 6, no. 7 (2018): 445-449. doi: 10.12691/jfnr-6-7-4.

\section{Introduction}

Pear thinning is a common agricultural practice worldwide that involves the removal of immature pear fruits from trees to ensure better harvest quality. These immature pears are usually treated as agricultural waste. However, immature pear fruits can contain high levels of beneficial polyphenols [1] and may have commercial utility.

Diabetes has received considerable attention owing to the increasing number of people diagnosed with this chronic disease [2]. Postprandial hyperglycemia is an important risk factor for the development of type II diabetes [3]. Controlling blood glucose levels is the most effective method for preventing diabetes deterioration and hyperglycemia [4]. All dietary carbohydrates are hydrolyzed by enzymes to yield simple sugars, which can improve blood glucose levels [5]. $\alpha$-Glucosidase is an intestinal cell membrane enzyme that can hydrolyze polysaccharides; hence, inhibiting the activity of $\alpha$-glucosidase may be an effective way to treat pre-diabetes and slow the progression of diabetes [6].

Several synthetic $\alpha$-glucosidase inhibitors, such as acarbose and voglibose, have been developed, but these exhibit various side effects, such as digestion disorders, flatulence, and liver function disorders [7]. Recently, many studies have focused on natural $\alpha$-glucosidase inhibitors from plants. Although it has been reported that polyphenols from fruits [8,9] may inhibit $\alpha$-glucosidase activity, few studies have examined the inhibitory activity of the polyphenols from immature pear fruits against both $\alpha$-glucosidase and blood glucose levels.

In the present study, we utilized pear fruits from the Hosui and Kosui cultivars (Pyrus pyrifolia), which are the major varieties in Japan. We examined the inhibitory effects of polyphenols from the immature pear fruits of these cultivars on $\alpha$-glucosidase activity in vitro and blood 
glucose levels in mice after the oral administration of starch.

\section{Materials and Methods}

\subsection{Materials}

Immature pear fruits of the Hosui and Kosui cultivars were obtained from Yamamoto Orchard in the town of Yoichi (Hokkaido, Japan). For each immature pear fruit, the diameter was approximately $2 \mathrm{~cm}$, moisture content was $82 \%$, and weight ranged from $42.1 \mathrm{~g}$ to $43.3 \mathrm{~g}$. The immature pear fruits were harvested approximately 70 days after flowering. Diaion HP-20 columns and Sephadex LH-20 columns for chromatography were obtained from the Mitsubishi Chemical Corporation (Tokyo, Japan) and GE Healthcare Bio-Sciences AB (Uppsala, Sweden), respectively. All other reagents and chemicals were purchased from Wako Pure Chemical Industries, Ltd. (Osaka, Japan), unless otherwise stated.

\subsection{Extract Preparation and Fractionation}

Immature pear fruits were freeze-dried for 3 days and then crushed by blender, followed by extraction with 20 $\mathrm{mL}$ of $80 \%$ ethanol. After ultrasound treatment for $30 \mathrm{~min}$, the mixture was centrifuged at $1,006 \times g$ for $10 \mathrm{~min}$ to obtain the supernatant. The same extraction process was repeated twice more. The residues were subjected to another three rounds of extraction with $70 \%$ acetone-water, and the supernatant was obtained. Then, the supernatant was mixed, concentrated by rotary evaporation in a vacuum, and purified by chromatography through a Diaion HP-20 column. The columns were washed with distilled water and then eluted with methanol. The methanol solution was concentrated by rotary evaporation in a vacuum and dissolved in $2 \mathrm{~mL}$ of methanol for the experiment. Part of the concentrate was dissolved in ethanol and fractionated by Sephadex LH-20 column chromatography. The column was successively eluted with ethanol, methanol, and 60\% acetone to collect fraction I (Fra.I), fraction II (Fra.II), and fraction III (Fra.III), respectively.

\subsection{Quantification of Polyphenols}

Polyphenols were quantified using the Folin-Ciocalteu method [10]. The methanol fraction (HP-20 column; $100 \mu \mathrm{L}$ ) was treated with $300 \mu \mathrm{L}$ of distilled water, $400 \mu \mathrm{L}$ of Folin-Ciocalteu reagent, and $400 \mu \mathrm{L}$ of a $10 \%$ $\mathrm{Na}_{2} \mathrm{CO}_{3}$ solution. The mixture was prepared in triplicate, incubated at $30{ }^{\circ} \mathrm{C}$ for $30 \mathrm{~min}$, and centrifuged at $1,006 \times \mathrm{g}$ for $10 \mathrm{~min}$. The absorbance of the mixed supernatant was measured at $760 \mathrm{~nm}$. The polyphenol content is expressed in milligrams of catechin equivalents per gram of dry weight of immature pear fruits (mg/g D.W.).

\subsection{Estimation of 2,2-diphenyl-1- picrylhydrazyl (DPPH) Radical Scavenging Activity}

DPPH radical scavenging activity was evaluated by the method described by Brand-Williams et al. [11] with some modifications. A $50-\mu \mathrm{L}$ aliquot of the methanol fraction (HP-20 column) was mixed with $100 \mu \mathrm{L}$ of ethanol, and the mixture was supplemented with $150 \mu \mathrm{L}$ of $0.5 \mathrm{mM}$ $\mathrm{DPPH}$ in ethanol. The absorbance of the mixture was measured using a microplate reader at $517 \mathrm{~nm}$. The DPPH radical scavenging activity is expressed in micromoles of trolox equivalents per gram of dry weight of immature pear fruits ( $\mu \mathrm{mol} / \mathrm{g}$ D.W.).

\section{5. $\alpha$-Glucosidase Inhibitory Activity}

$\alpha$-Glucosidase inhibition was analyzed following the methods of Matsumoto et al. [12] with modifications. Sucrose was broken down by $\alpha$-glucosidase, and the amount of reducing sugar was calculated based on the $\alpha$-glucose content. In total, $0.8 \mathrm{~mL}$ of enzyme reaction solution [ $50 \mu \mathrm{L}$ of $0.4 \%$ sucrose, $625 \mu \mathrm{L}$ of $0.1 \mathrm{~mol} / \mathrm{L}$ sodium phosphate buffer ( $\mathrm{pH} 6.8)$, and $125 \mu \mathrm{L}$ of $1 \%$ $\mathrm{NaCl}$ ] was pre-incubated at $37^{\circ} \mathrm{C}$ for $30 \mathrm{~min}$. The methanol fraction (HP-20 column) was concentrated by rotary evaporation in a vacuum and dissolved in distilled water. The distilled water fraction (polyphenol concentration, $0-200 \mu \mathrm{g} / \mathrm{mL}$ ) was added to $0.1 \mathrm{U} / \mathrm{mL} \alpha$-glucosidase solution (EC3.2.1.20; Oriental Yeast Co., Ltd., Tokyo, Japan) at $37^{\circ} \mathrm{C}$ for $10 \mathrm{~min}$. After pre-incubation, $200 \mu \mathrm{L}$ of the mixture (polyphenol extract and $\alpha$-glucosidase) was added to the enzyme reaction solution and incubated at $37^{\circ} \mathrm{C}$ for $30 \mathrm{~min}$. The reaction was terminated by adding $125 \mathrm{~mL}$ of $2 \mathrm{M} \mathrm{NaOH}$ and 1\% dinitrosalicylic acid in boiling water for $10 \mathrm{~min}$. After incubation, the mixture was analyzed at $540 \mathrm{~nm}$ at $25^{\circ} \mathrm{C}$. Enzyme inhibitory reactions for all polyphenol extract concentrations were replicated three times. The $\alpha$-glucosidase inhibitory activity is expressed as the percent inhibition. The concentration of inhibitors required for the inhibition of $50 \%$ of the enzyme activity under the assay conditions was defined as the $\mathrm{IC}_{50}$ value.

\subsection{Oral Starch Tolerance Test in Mice}

Male DDY mice (Japan SLC, Inc., Shizuoka, Japan) were housed in plastic cages at $23{ }^{\circ} \mathrm{C}$ under a $12 \mathrm{~h} / 12 \mathrm{~h}$ light/dark cycle and a relative humidity of $60 \%$. Mice had free access to feed (CE-2; CLEA Japan, Inc., Tokyo, Japan) and water for 1 week before the experiment. The feed contained $344.9 \mathrm{kcal} / 100 \mathrm{~g}$ and was composed of (w/w \%) $24.9 \%$ crude protein, $4.6 \%$ crude fat, $4.1 \%$ crude fiber, $6.6 \%$ crude ash, $51.0 \%$ NFE (nitrogen-free extract), and $8.9 \%$ moisture. After 1 week, the animals were randomly divided into experimental groups (8 mice per group). The mice were fasted for $16 \mathrm{~h}$, and blood was drawn from the tail vein and subjected to assays to determine blood glucose levels. The polyphenols from the immature Hosui fruits were suspended in physiological solution and were administered in doses of 250 and $500 \mathrm{mg} / \mathrm{kg}$. After the oral administration of the suspension of polyphenol compounds for $30 \mathrm{~min}$, a single oral injection of $2 \mathrm{~g} / \mathrm{kg}$ starch in physiological solution was administered. Blood was withdrawn from the tail vein at $0.5,1$, and $2 \mathrm{~h}$, and blood glucose levels were analyzed using the OMRON Precision Exceed HEA-216 (Omron Healthcare Co., Ltd., Kyoto, Japan) according to the manufacturer's instructions. This study was approved by the regulatory authority of the National University Corporation Obihiro 
University of Agriculture and Veterinary Medicine, and it adhered to the standard principles described in the Guide for the Care and Use of Laboratory Animals.

\subsection{Statistical Analysis}

Values are presented as the means \pm standard error of 5 out of 8 mice in each group. Statistical significance was evaluated by analysis of variance (ANOVA) and least significant difference (LSD) tests (SAS Enterprise Guide 5.1 system, Cary, NC, USA). Differences were considered significant at $p<0.05$.

\section{Results and Discussion}

\subsection{Polyphenol Content, DPPH Radical Scavenging Activity, and Polyphenol Fractions}

The polyphenol content of the immature Hosui pear fruits was higher than that of the immature Kosui pear fruits, and the same pattern was observed for the DPPH radical scavenging activities of the two types of fruits (Table 1). Thus, it appears that the polyphenols from both cultivars exhibited antioxidant activity. Similarly, it has been reported that the polyphenols from grape, apple, cacao, and persimmon exhibit antioxidant activity [13].

Table 1. Polyphenol content, DPPH radical scavenging activity, and a-glucosidase inhibitory activity in immature Hosui and Kosui pear fruits

\begin{tabular}{cccc}
\hline Cultivar & Polyphenols & $\begin{array}{c}\text { DPPH radical } \\
\text { scavenging activity } \\
(\mu \mathrm{mol} / \mathrm{g} \mathrm{D} . W .)\end{array}$ & $\begin{array}{c}\alpha \text {-Glucosidase } \\
\text { inhibitory activity } \\
\text { IC }_{50}(\mu \mathrm{g} / \mathrm{mL})\end{array}$ \\
\hline Hosui & $28.5^{\mathrm{a}} \pm 0.6$ & $115.8^{\mathrm{a}} \pm 1.6$ & 21.3 \\
Kosui & $20.9^{\mathrm{b}} \pm 0.3$ & $76.3^{\mathrm{b}} \pm 5.8$ & 66.4 \\
\hline
\end{tabular}

D.W.: dry weight of immature pear fruits. Values represent mean \pm S.E.M. Different superscript letters indicate significant differences $(p<0.05)$.

We performed Sephadex LH-20 column chromatography to obtain three polyphenol fractions, i.e., Fra.I, Fra.II, and Fra.III, from the immature Hosui and Kosui pear fruits (Figure 1). The polyphenol contents in Fra.I, Fra.II, and Fra.III from Hosui immature pear fruits accounted for 6\%, $63 \%$, and $31 \%$ of the total weight, respectively,

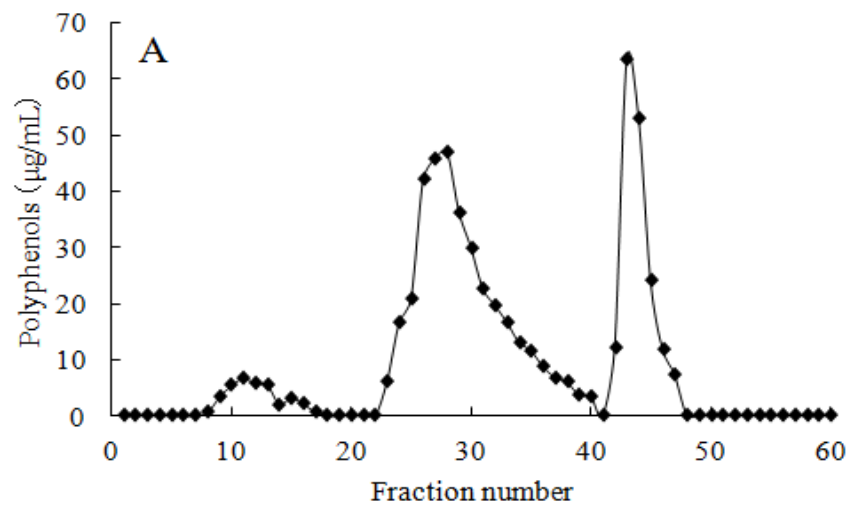

while those from Kosui fruits accounted for 24\%, 55\%, and $21 \%$, respectively. Thus, both Hosui and Kosui fruits had higher percentages of Fra.II than of the other fractions. According to Saito et al. [14], Fra.I contains monomeric polyphenols, Fra.II contains oligomeric polyphenols, and Fra.III contains polymeric polyphenols. Based on our results, the total amounts of non-monomeric (oligomeric and polymeric) polyphenols were $94 \%$ and $76 \%$ in immature Hosui and Kosui fruits, respectively. While the monomeric polyphenols arbutin and chlorogenic acid have been shown to be major polyphenols related to DPPH radical scavenging activity in Japanese pears [15], in our study, oligomeric and polymeric polyphenols were the major polyphenols in immature Hosui and Kosui pear fruits. Thus, we speculate that the DPPH radical scavenging activity of these polyphenols are mainly related to non-monomeric polyphenols. Notably, we previously reported that polymeric polyphenols may possess greater antioxidant activity [16].

\section{2. $\alpha$-Glucosidase Inhibitory Activity}

We next analyzed the inhibitory effects of the polyphenols on $\alpha$-glucosidase. Polyphenols from the Hosui and Kosui immature pear fruits inhibited the activity of $\alpha$-glucosidase in a dose-dependent manner. Several studies have shown the $\alpha$-glucosidase inhibitory activity of various fruits and vegetables $[8,9]$. The $\mathrm{IC}_{50}$ value of the polyphenols from immature Hosui fruits was $21.3 \mu \mathrm{g} / \mathrm{mL}$, and that of the polyphenols from Kosui fruits was $66.4 \mu \mathrm{g} / \mathrm{mL}$, indicating that the former exhibited greater $\alpha$-glucosidase inhibitory activity (Table 1). Moreover, we analyzed and compared the inhibitory effects of $20 \mu \mathrm{g} / \mathrm{mL}$ of polyphenols from Fra.I, Fra.II, and Fra.III on $\alpha$-glucosidase (Figure 2). No inhibitory activity was detected in Fra.I. The rates of inhibition of $\alpha$-glucosidase were $14.9 \%$ and $11.7 \%$ for Fra.II polyphenols derived from Hosui and Kosui fruits, respectively, and $18.5 \%$ and $15.7 \%$ for Fra.III polyphenols, respectively; thus, Fra.III showed more potent $\alpha$ glucosidase inhibitory activity. We speculate that higher degrees of polymerization in polyphenols results in stronger inhibition of $\alpha$-glucosidase. Consistent with this, we also found that oligomeric and polymeric polyphenols exhibited stronger $\alpha$-glucosidase inhibitory activity than monomeric phenolic compounds in scarlet runner beans [17].

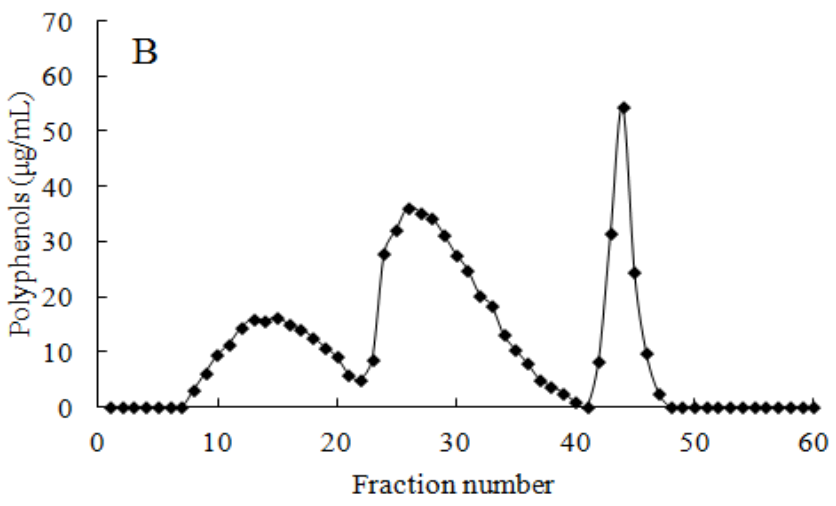

Figure 1. Sephadex LH-20 column chromatograms of polyphenols prepared from immature Hosui and Kosui pear fruits. The polyphenol fractions obtained by Diaion HP-20 column chromatography were applied to Sephadex LH-20 column chromatography and eluted with ethanol, fraction number 1-20 (Fra.I); methanol, fraction number 21-40, (Fra.II); and 60\% acetone, fraction number 41-60, (Fra.III). (A) Immature Hosui pear fruits. (B) Immature Kosui pear fruits 

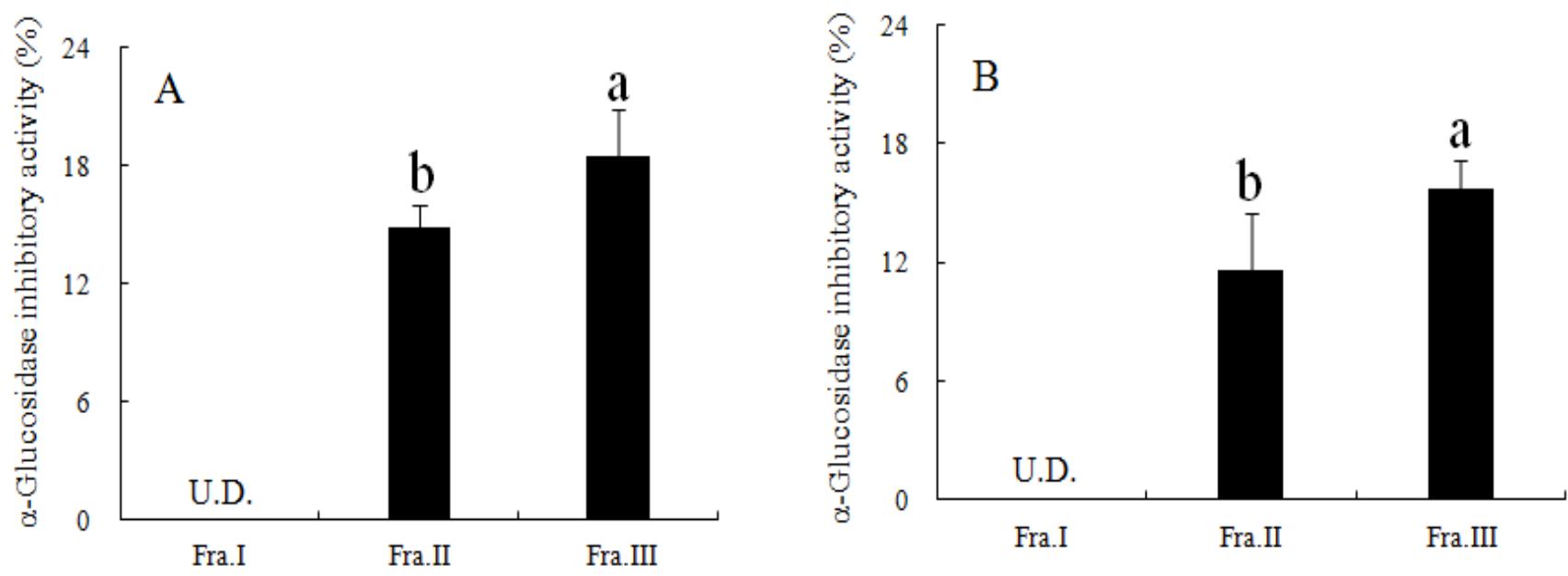

Figure 2. $\alpha$-Glucosidase inhibitory activity of $20 \mu \mathrm{g} / \mathrm{mL}$ of Fra.I, Fra.II, or Fra.III polyphenols from immature Hosui and Kosui pear fruits. (A) Immature Hosui pear fruits. (B) Immature Kosui pear fruits. U.D., undetected. Values represent mean \pm S.E.M. Different superscript letters indicate significant differences $(p<0.05)$

\subsection{Inhibitory Effects of Polyphenols on Blood Glucose Levels after Oral Administration of Starch}

Compared with those in immature Kosui fruits, the polyphenols of immature Hosui fruits resulted in more effective inhibition of $\alpha$-glucosidase in vitro. Therefore, the polyphenols from immature Hosui fruits were tested for their inhibitory effects on blood glucose levels by conducting an oral starch tolerance test in mice. After the administration of starch, the maximum increase in blood glucose levels was observed at $30 \mathrm{~min}$ in all mice, but mice treated with $250 \mathrm{mg} / \mathrm{kg}$ or $500 \mathrm{mg} / \mathrm{kg}$ mouse exhibited significantly lower blood glucose concentrations than those in the control group ( $p<0.05$; Figure 3A). At 60 and $120 \mathrm{~min}$, the $500 \mathrm{mg} / \mathrm{kg}$ mouse group still showed significantly lower glucose levels than the control group $(p<0.05)$, indicating that Hosui fruit polyphenols suppress the breakdown of starch into glucose. As immature Kosui

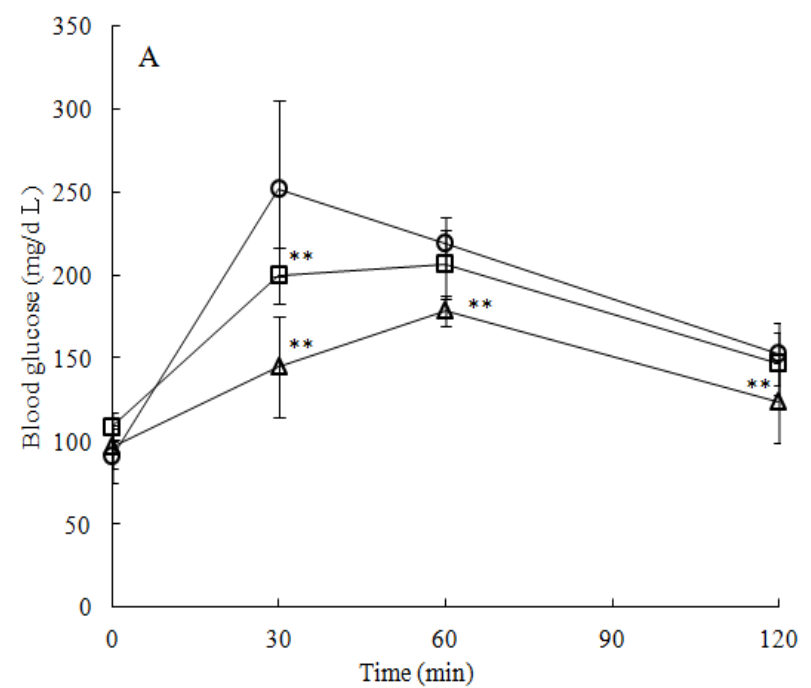

fruits shared the same polyphenol distribution as immature Hosui fruits and also exhibited $\alpha$-glucosidase inhibitory activity, we speculate that immature Kosui fruits would also effectively reduce blood glucose levels in vivo, though much higher concentrations of polyphenols would be needed due to their less potent activity. The area under the curve (AUC) of blood glucose levels over the 120-min period is shown in Figure 3B. The AUC was significantly lower for the $500 \mathrm{mg} / \mathrm{kg}$ mouse group compared with that of the control group, confirming that an increase in the glucose level was significantly suppressed by the polyphenols from immature Hosui fruits. This is consistent with results from Wang et al. 2015 [18], who reported that Yaguang pear (Pyrus ussuriensis) fruits exhibited potential $\alpha$-glucosidase inhibitory activity in vitro and exerted antidiabetic effects in streptozocin-induced type 2 diabetic mice. Moreover, the polyphenols from the fruits of grape, apple, cacao, and persimmon have also been found to suppress increases in blood glucose [19,20,21,22].

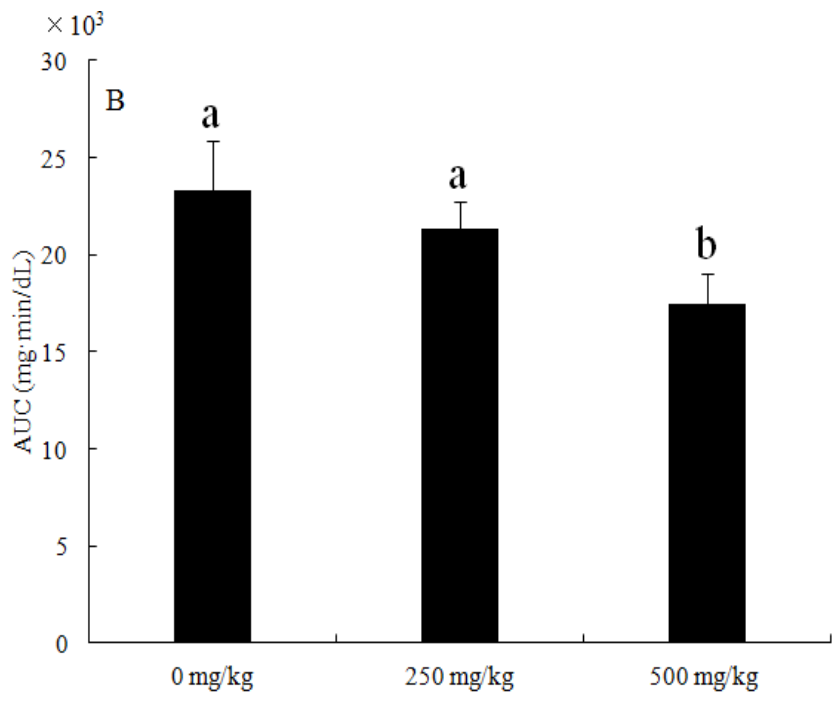

Figure 3. Inhibitory effect of immature Hosui pear fruit polyphenols on blood glucose levels in vivo. (A) Blood glucose levels after oral starch tolerance test in mice to monitor the inhibitory effect of polyphenols from immature Hosui pear fruits. Circles, $0 \mathrm{mg} / \mathrm{kg} \mathrm{mouse}$; squares, $250 \mathrm{mg} / \mathrm{kg}$ mouse; triangles, $500 \mathrm{mg} / \mathrm{kg}$ mouse. Data represent the mean \pm standard error of 5 mice in each group. ${ }^{* *} p<0.05$ vs. starch (control). (B) AUC values of data shown in panel A. Values represent mean \pm S.E.M. Different superscript letters indicate significant differences $(p<0.05)$ 


\section{Conclusions}

In this study, we found that immature Hosui fruits possess a higher polyphenol content and DPPH radical scavenging activity than immature Kosui fruits. The immature pear fruits of the Hosui and Kosui cultivars showed high ratios of oligomeric and polymeric polyphenols, which inhibited the activity of $\alpha$-glucosidase in a dose-dependent manner. Moreover, the polyphenols from immature Hosui fruits effectively suppressed elevations in blood glucose levels in vivo, as shown by experiments involving the oral administration of starch in mice. These observations indicate that Hosui and Kosui immature pear fruits, which are usually treated as agricultural waste, may serve as a source of nutraceuticals with anti-diabetic activity.

\section{Acknowledgements}

We acknowledge the financial support provided by the Obihiro University of Agriculture and Veterinary Medicine, as well as Iwate University. Moreover, we thank Yamamoto Orchard (Yoichi, Hokkaido) for providing the immature pear fruits used in this study.

\section{Statement of Competing Interests}

The authors have no competing interests.

\section{References}

[1] Heng, M.Y., Katayama, S., Mitani, T., Ong, E.S. and Nakamura, S, "Solventless extraction methods for immature fruits: Evaluation of their antioxidant and cytoprotective activities," Food Chemistry, 221. 1388-1393. April 2017.

[2] Cohen, P. and Goedert, M, "GSK3 inhibitors: development and therapeutic potential," Nature Reviews Drug Discovery, 3 (6). 479-487. June 2004.

[3] Dong, H.Q., Li, M., Zhu, F., Liu, F.L. and Huang, J.B, “Inhibitory potential of trilobatin from Lithocarpus polystachyus Rehd against $\alpha$-glucosidase and $\alpha$-amylase linked to type 2 diabetes,” Food Chemistry, 130. 261-266. January 2012.

[4] Mertes, G, "Safety and efficacy of acarbose in the treatment of type 2 diabetes: Data from a 5-year surveillance study,” Diabetes Research and Clinical Practice, 52. 193-204. June 2001.

[5] Xiao, H.Z., Liu, B.G., Mo, H.Z. and Liang, G.Z, "Comparative evaluation of tannic acid inhibiting $\alpha$-glucosidase and trypsin," Food Research International, 76. 605-610. October 2015.

[6] Baron, A.D, "Postprandial hyperglycaemia and alpha-glucosidase inhibitors," Diabetes Research and Clinical Practice, 40. 51-55. July 1998.

[7] Hollander, P, "Safety profile of acarbose, an alpha-glucosidase inhibitor,” Drugs, 44 (Suppl. 3). 47-53. 1992.
[8] Kawada, Y., Miura, M. and Gomyo, T, "Inhibitory effect of vegetables, fruits and herbs on alpha-glucosidase in an immobilized enzyme assay system," Food Science and Technology Research, 12. 275-277. 2006.

[9] Koga, K., Shibata, H., Yoshino, K. and Nomoto, K, "Effects of $50 \%$ ethanol extract from rosemary (Rosmarinus officinalis) on alpha-glucosidase inhibitory activity and the elevation of plasma glucose level in rats, and its active compound," Journal of Food Science, 71. S507-S512, 2006.

[10] Miyashita, J., Nishi S., Saito Y., Koaze, H., Hironaka, K. and Kojima, M, "Annual variations in the anthocyanin contents of blueberry fruit grown in Hokkaido," Research Bulletin of Obihiro University of Agriculture and Veterinary Medicine, 28. 35-40. 2007.

[11] Brand-Williams, W., Cuvelier, M.E. and Berset, C, "Use of a free radical method to evaluate antioxidant activity," LebensmittelWissenschaft und Technologie, 28. 25-30. 1995.

[12] Matsumoto, N., Ishigaki, A., Iwashina, H. and Hara, Y, "Reduction of blood glucose levels by tea catechin," Bioscience, Biotechnology, Biochemistry, 57. 525-527. 1993.

[13] Koga, T., Moro, K., Nakamori, K., Yamakoshi, J., Hosoyama, H., Kataoka, S. and Ariga, T, "Increase of antioxidative potential of rat plasma by oral administration of proanthocyanidin-rich extract from grape seeds,” Journal of Agricultural and Food Chemistry, 47. 1892-1897. May 1999.

[14] Saito, Y., Nishi, S., Koaze, H., Hironaka, K. and Kojima, M, "Antioxidant and inhibitory activity on $\alpha$-amylase and $\alpha$ glucosidase in legume polyphenols," Nippon Shokuhin Kagaku Kogaku Kaishi, 54 (12). 563-567. 2007.

[15] Sugawara, T. and Igarashi, K, "Variation in polyphenol components and radical scavenging activity of Japanese pear (Pyrus serotina Rehder var. culta Rehder) during fruit maturation," Nippon Shokuhin Kagaku Kogaku Kaishi, 60 (9). 516-520. 2013.

[16] Ci, Z.H., Jiang, C.Y., Tsukamoto, C. and Kojima, M, "DPPH radical scavenging activity and polyphenols in the pods of 3 common beans," Journal of Food and Nutrition Research, 5 (12). 900-907. November 2017.

[17] Ci, Z.H, Jiang, C.Y. and Kojima, M, "Suppressive effect of polyphenols from the seed coat of scarlet runner beans on blood glucose levels," Journal of Food and Nutrition Research, 6 (3). 182-186. 2018.

[18] Wang, T.T., Li, X., Zhou, B., Li, H.F., Zeng, J. and Gao, W.Y, "Anti-diabetic activity in type 2 diabetic mice and $\alpha$-glucosidase inhibitory, antioxidant and anti-inflammatory potential of chemically profiled pear peel and pulp extracts (Pyrus spp.)," Journal of Functional Foods, 13. 276-288. 2015.

[19] Kometani, T. and Takemori, K, "Polyphenols from persimmon fruits as a functional foods," Nippon Shokuhin Kagaku Kogaku Kaishi, 63 (7). 331-337. 2016

[20] Schulze, C., Bangert, A., Kottra, G., Geillinger, K.E., Schwanck, B., Vollert, H., Blaschek, W. and Daniel, H, "Inhibition of the intestinal sodium-coupled glucose transporter 1 (SGLT1) by extracts and polyphenols from apple reduces postprandial blood glucose levels in mice and humans," Molecular Nutrition \& Food Research, 58. 1795-1808. September 2014.

[21] Yamashita, Y., Okabe, M., Natsume, M. and Ashida, H, "Prevention mechanisms of glucose intolerance and obesity by cacao liquor procyanidin extract in high-fat diet-fed C57BL/6 mice," Archives of Biochemistry and Biophysics, 527. 95-104. November 2012.

[22] Grassi, D., Lippi, C., Necozione, S., Desideri, G. and Ferri, C, "Short-term administration of dark chocolate is followed by a significant increase in insulin sensitivity and a decrease in blood pressure in healthy persons," American Journal of Clinical Nutrition, 81. 611-614. March 2004. 